\title{
Cavity lining in primary teeth
}

\section{Abstracted from}

\section{Schwendicke F, Göstemeyer G, Gluud C.}

Cavity lining after excavating caries lesions: meta-analysis and trial sequential analysis of randomized clinical trials. J Den 2015; 43: 1291-1297.

Address for correspondence: Falk Schwendicke, Department for Operative and Conservative Dentistry, Charité-Universitätsmedizin Berlin, Aßmannshauser Str. 4-6, 14197 Berlin, Germany.

Fax: +4930450 762556. E-mail: falk.schwendicke@charite.de

\section{Question: What are the benefits of cavity lining?}

Data sources Embase, Medline, Cochrane Central, Biomed Central and Open Grey databases and bibliographies of identified studies. Study selection Randomised controlled trials investigating humans with primary caries lesions receiving operative treatment involving caries removal and restoration, with minimum two treatment groups comparing different cavity treatments before restoration (no lining versus lining) were included.

Data extraction and synthesis Data were extracted independently by two reviewers and study quality assessed using the Cochrane risk of bias tool. Random effect meta-analysis was carried out.

Results Three studies involving a total of 89 patients were included. All the studies involved primary teeth and were conducted in Brazil. Follow-up periods ranged from 26-53 months. All the studies were considered to be at high risk of bias. Restoring the cavity without lining did not significantly affect the risk of failure. The quality of the evidence was low.

Conclusions Current evidence does not support strong recommendations to use or not to use liners after caries removal and before restoring cavities. Our findings are restricted to primary teeth after selective excavation, with only one liner (calcium hydroxide) being used for comparison.

\section{Commentary}

The application of cavity liners before restoring deep carious lesions has been widely practised over the last few decades. ${ }^{1}$ Cavity liners have been defined as 'materials that are applied as a thin layer to seal the dentine floor and walls of the cavity from the influx of bacteria and irritants from restorative procedures. ${ }^{2}$ These materials aim to encourage the development of reactionary dentine and remineralisation. ${ }^{1}$ More recently, stepwise and partial caries excavation has challenged the traditional method of complete caries removal followed by the application of a cavity liner. $3,4,5$

The authors of this study aimed to evaluate the risk of failure with (control group) or without (experimental group) the use of a cavity liner following the removal of primary carious lesions after at least one-year. A comprehensive search strategy was used to identify randomised clinical trials. The authors gave an account of the search terms and Boolean operators used. A flow chart showing the number of studies returned was also shown. The screening of grey literature, hand searching, contacting authors and placing no language or date restrictions added to the robustness of the search.

Two reviewers assessed titles and abstracts together with risk of bias as outlined by the Cochrane Collaboration. The level of agreement between reviewers was not given. Data from three studies with a combined total of 130 carious lesions in primary teeth in 89 patients with a follow-up from 36 to 53 months were included. The included studies were carried out in Brazil by one team in a secondary care setting. They were all considered to have a high risk of bias, which should raise doubts about the applicability of this study. Calcium hydroxide was the liner of choice in all three studies. Gutta percha was used as a placebo liner in one of the included studies. All teeth were subsequently restored with composite resin under rubber dam. Yearly drop outs ranged from between 5 to $12 \%$.

The authors used an intention-to-treat and per-protocol analysis to account for possible attrition bias. No statistically significant differences were found between the control and experimental groups. The results indicated fewer failures in the group without a lining when using both the intention-to-treat $(\mathrm{RR}=0.71(95 \% \mathrm{CI}$ $0.49-1.04)$ and per-protocol analyses $(\mathrm{RR}=0.52(95 \% \mathrm{CI} 0.24-$ 1.10). Acid etching and dentine bonding agents may have negated the potential benefits of using calcium hydroxide. ${ }^{6}$ The use of calcium hydroxide would have also reduced the area for bonding.

The authors conclude that their findings cannot be used to 'recommend or refute the use of calcium hydroxide after caries 
removal' as the included studies have been restricted to primary teeth after selective excavation of caries and the use of only one liner (calcium hydroxide). Confounding factors such as the restorative material, bonding procedure, operator, age of patient, caries risk and extent of restoration should be taken into account. They can all influence the risk of failure.

It is the remaining dentine thickness following cavity preparation that has a greater influence on odontoblast survival and dentine repair activity than the restorative material or liner used. ${ }^{7}$ The preservation of dentine should be the primary focus rather than the choice of lining material when managing deep carious lesions. Dentists may, therefore, need to appraise their management of deep carious lesions and the use of cavity liners with increasing evidence to support a minimally invasive approach. . $^{3,4,5}$

Ravi Chauhan

Restorative Dentistry, King's College Dental Hospital and William Harvey Hospital (Kent, Surrey and Sussex Deanery), UK

\section{Practice points}

- Acid etching and dentine bonding agents can adversely affect the properties of calcium hydroxide.

- The patients' caries risk and motivation should be taken into account when electing to use a minimally invasive approach.

1. Weiner R. Liners and bases in general dentistry. Aust Dent J 2011; 56: 11-22.

2. Ferracane JL. Materials in dentistry: principles and applications. 2nd edn. Philadelphia: Lippincott Williams and Wilkins, 2001.

3. Ricketts $D$, Lamont $T$, Innes NP, Kidd E, Clarkson JE. Operative caries management in adults and children. Cochrane Database Syst Rev 2013; 3: CD003808.

4. Thompson V, Craig RG, Curro FA, Green WS, Ship JA. Treatment of deep carious lesions by complete excavation or partial removal: a critical review. J Am Dent Assoc 2008; 139: 705-712.

5. Bjørndal L, Reit C, Bruun $\mathrm{G}$, et al. Treatment of deep caries lesions in adults: randomized clinical trials comparing stepwise vs. direct complete excavation, and direct pulp capping vs. partial pulpotomy. Eur J Oral Sci 2010; 118: 290-297.

6. El-Araby A, Al-Jabab A. The effect of some dentin bonding agents on Dycal lining cement. Saudi Dent / 2004; 16: 102-106.

7. About I, Murray PE, Franquin JC, Remusat M, Smith AJ. The effect of cavity restoration variables on odontoblast cell numbers and dental repair. J Dent 2001; 29: 109-117.

Evidence-Based Dentistry (2016) 17, 17-18. doi:10.1038/sj.ebd.6401151 